$\Rightarrow$ HUMAN GENOMICS

\title{
The finer points of human recombination
}

Indications of heritable variation in patterns and rates of human recombination have generated great interest. Two new studies take advantage of high-density genotyping to provide a more detailed picture of this variation.

New platforms for genotyping large SNP sets allow the locations of recombination events to be identified at high resolution in pedigrees. Unlike previous large-scale studies, which relied on the indirect estimation of recombination from linkage disequilibrium (LD), this method allows variation between individuals and sexes to be assessed. It also allows our relatively poor understanding of fine-scale rates of recombination in females to be addressed - many previous findings relied on sperm typing.

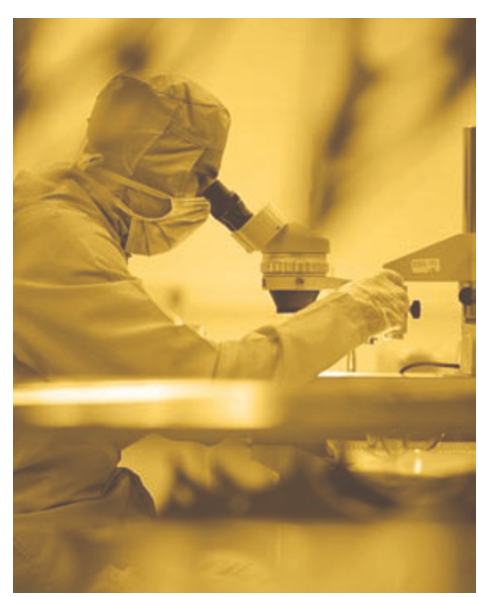

Using one large SNP set to look at recombination in 725 related individuals from the Hutterite population, Coop and colleagues estimated genome-wide recombination rates that closely match figures from previous studies. Furthermore, they confirmed - for both sexes - a high degree of inter-individual variation in the mean number of crossovers. Interestingly, the chromosomes for which there is marked variation in recombination rates were different in males and females, suggesting sex-specific effects.

The same group also looked at the distribution of recombination hot spots - sites of high historical recombination rates identified from LD studies. The authors showed that although most of these hot spots are used in both sexes, some are much more active in one sex. Excitingly, they also found a high level of variation among individuals, both male and female, in terms of the fraction of recombination events that take place in these hot spots, suggesting that there are heritable differences in the recombination machinery.

In a second study, Kong and colleagues mapped genetic variants that underlie variability in recombination rates. They started with a genome-wide association study in 1,887 fathers and 1,702 mothers, and then carried out a follow-up study that included 4,388 nuclear families and 19,578 genotyped individuals. From these studies, three SNPs were identified that are associated with recombination rates in both sexes. Intriguingly, however, their effects on recombination were in the opposite direction in males and females. Similarly, a haplotype containing the pair of SNPs that correlates with the highest recombination rate in males is associated with a low rate in females. Tantalizingly, although the functional significance of the variants has yet to be confirmed, they map to $\underline{R N F 212}$ - a gene for which the putative worm orthologue is involved in recombination.

These findings should spark increased interest in the extent and basis of variation in recombination patterns within human populations, given the important implications that such results have for understanding human genetic diversity and genome evolution.

Louisa Flintoft

ORIGINAL RESEARCH PAPERS

Coop, G., Wen, X., Ober, C., Pritchard, J. K. \& Przeworski, M. High-resolution mapping of crossovers reveals extensive variation in finescale recombination patterns among humans. Science 31 Jan 2008 (doi:10.1126/ science.1151851)|Kong, A. et al. Sequence variants in the RNF212 gene associate with genomewide recombination rate. Science 31 Jan 2008 (doi:10.1126/science.1152422) FURTHER READINC

Coop, G. \& Przeworski, M. An evolutionary view of human recombination. Nature Rev. Genet. 8 , 23-34 (2006) 